\title{
Efficacy of ciclesonide in the treatment of patients with asthma exacerbation
}

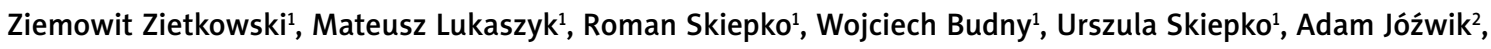 \\ Anna Bodzenta-Lukaszyk ${ }^{1}$
}

${ }^{1}$ Department of Allergology and Internal Medicine, Medical University of Bialystok, Bialystok, Poland ${ }^{2}$ Institute of Biocybernetics and Biomedical Engineering, Polish Academy of Sciences, Warsaw, Poland

Adv Dermatol Allergol 2019; XXXVI (2): 217-222

DOI: https://doi.org/10.5114/ada.2019.84596

\begin{abstract}
Introduction: Progressing deterioration of the lung function, dyspnoea, cough, wheezing and chest tightness are the main features of asthma exacerbations. The first step in the prevention of severe asthma exacerbations is to intensify the anti-inflammatory treatment with high doses of inhaled corticosteroids (ICS).

Aim: To assess the efficacy of ciclesonide in patients who have been losing control of asthma despite being treated with medium doses of inhaled corticosteroids and long-acting $\beta_{2}$-agonists (LABA) as the second controller. Material and methods: The study was conducted in a group of 74 asthmatic patients who have been losing control of their asthma. Subjects entering the study received the following anti-inflammatory interventions: high doses of ciclesonide $(1280 \mu \mathrm{g})$ or $640 \mu \mathrm{g}$ of ciclesonide added to a current dose of ICS or a doubled dose of current ICS. Results: Treatment options containing ciclesonide have shown statistically and clinically important advantages (improvement of Asthma Control Test score, reduction in rescue medication consumption, reduction in day and night symptoms score, improvement in spirometry parameters, decrease in exhaled nitric oxide, and no necessity of oral corticosteroids treatment) in comparison to patients for whom medium doses of the previously used inhaled corticosteroid were doubled.

Conclusions: Treating with high doses of ciclesonide is characterised by a quick and potent anti-inflammatory effect as well as prompt clinical improvement along with the proper safety profile in patients experiencing asthma exacerbations.
\end{abstract}

Key words: ciclesonide, asthma exacerbations, airway inflammation.

\section{Introduction}

Small airways play an important role in asthma as well as in its severe phenotype and during the exacerbations. This is proven by numerous publications [1-4]. In our previously published work we showed that extra-fine ciclesonide provides a more potent anti-inflammatory effect than standard particle inhaled corticosteroids (ICS) [5].

William van Aalderen published a study in which he showed that small-particle ICS cause better prevention of exacerbations (manifested as a lower number of hospitalizations and exacerbations) and better asthma control than standard size particle ICS. Small-particle ICS were no worse in preventing exacerbations than a step-up longacting $\beta_{2}$-agonists (LABA)/ICS therapy [6].

Similar results concerning adult asthmatic patients have been published by E. Israeli. Small particle ICS have similar efficacy in exacerbation prevention to LABA/ICS combination treatment in step-up therapy [7].
Small airways play an important role in unstable and severe asthma pathogenesis [8]. Extra-fine ICS seem to penetrate to peripheral airways and produce a more potent anti-inflammatory effect in patients with small airways involved $[9,10]$.

Despite a significant progress in diagnostics and treatment of asthmatic patients within the natural course of the disease, exacerbations may occur. Progressing deterioration of the lung function, dyspnoea, cough, wheezing and chest tightness are the main symptoms of the asthma exacerbation. The first step in the prevention of severe asthma exacerbations is the intensification of the anti-inflammatory treatment with high doses of inhaled corticosteroids [11].

\section{Aim}

The aim of the study was to assess the efficacy of extra-fine ciclesonide $(\mathrm{CIC})$ in patients who have been

Address for correspondence: Ziemowit Zietkowski MD, PhD, Department of Allergology and Internal Medicine, Medical University of Bialystok, 24 A Skłodowskiej St, 15-276 Poland, phone: +48 857468 523, e-mail: z.zietkowski@wp.pl Received: 21.02.2018, accepted: 26.03.2018. 
losing control of their asthma despite being treated with medium doses of standard size-particle inhaled corticosteroids and LABA as the second controller.

\section{Material and methods}

\section{Patients}

Seventy-four patients who had been losing control of their asthma and/or had had mild exacerbation were included. Those patients had been treated with $500 \mathrm{\mu g}$ of fluticasone or $640 \mu \mathrm{g}$ of budesonide per day until the beginning of the study. Clinical criteria for qualification included an increase in the frequency of day and night-time asthma symptoms, an increase in short acting $\beta$-agonist (SABA) use, a decrease in Asthma Control Test (ACT) score, a confirmation of clinical worsening of the disease proven by a decrease in the forced expiratory volume in $1 \mathrm{~s}\left(\mathrm{FEV}_{1}\right)$ value. Patients requiring antibiotic therapy or fulfilling the criteria of severe asthma exacerbation during the baseline visit were not included.

\section{Study schedule}

The study period was 14 days. The clinical assessment, $F_{E N O}$ and spirometry were performed on day 1, 7 and 14 of the study. The clinical evaluation included: Asthma Control Test, day ( $0-5$ points) and night ( $0-4$ points) symptoms score, rescue medication consumption (salbutamol $100 \mu \mathrm{g}$ MDI), the necessity of oral corticosteroids (OCS) or antibiotics treatment, adverse events (AE) and resolution of asthma exacerbation (Table 1).

Subjects received the following anti-inflammatory interventions: high doses of CIC $1280 \mu g$ (group A), CIC $640 \mu g$ added to the current dose of ICS (group B) or a doubled dose of current ICS (group C). The LABA were continued in the same dose, SABA were used as a rescue medication.

This was an open study, performed without randomization. Patients were consecutively assigned to each of the study arms.

Asthma was diagnosed according to the criteria recommended by the GINA [11]. Asthma patients were nonsmokers and during the last year were not exposed to passive smoking.
The study protocol was approved by the Ethics and Research Committee of the Medical University of Bialystok, number R-I-002/455/2014. Informed consent form was obtained from every patient entering the study.

\section{Measurements}

Exhaled nitric oxide $\left(\mathrm{F}_{\mathrm{ENO}}\right)$ was measured with Sievers 280i NO Analyzer (Boulder, Colorado, USA), which uses a chemiluminescence technique. The measurements were performed at an expiratory flow of $50 \mathrm{ml} / \mathrm{s}$ according to ATS recommendations for on-line measurement of $F_{E N O}$ in adults [12].

The spirometry $\left(\mathrm{FEV}_{1}\right)$ was performed using a MasterScreen Pneumo PC spirometer (Jaeger, Hoechberg, Germany), according to the ATS standards [13].

The study was conducted between September 2014 and September 2015.

\section{Statistical analysis}

An analysed data set contained qualitative and quantitative variables that were not normally distributed. For this reason only nonparametric tests that do not require Gaussian data distribution were applied. The computational tasks consisted of an analysis of the dependence between two qualitative variables, an analysis of two quantitative variables, and comparison of independent and dependent groups described by the quantitative data. The relations between qualitative variables were analysed using the $\chi^{2}$ test based on frequency tables. An investigation of the dependence between quantitative variables was performed using the Spearman correlation rank test. Independent groups of patients were compared using the Kruskal-Wallis ANOVA and the MannWhitney tests, groups 3 and 2, respectively. An analysis of dependent groups of patients was performed through the application of the Friedman ANOVA test. All calculations were performed using the Statistica 9 package.

\section{Results}

Characteristics of patients are presented in Table 2. We did not observe any significant differences in patient

Table 1. The scheme of the protocol

\begin{tabular}{lll}
\hline Day 1 & Day 7 & Day 14 \\
\hline Clinical evaluation & Clinical evaluation, AE evaluation & Clinical evaluation, AE evaluation \\
FEV $_{1}$, FENO & FEV $_{1}$, FENO & FEV $_{1}$, FENO \\
\hline Anti-inflammatory interventions: & Other medical interventions: OCS, & Other medical interventions: OCS, \\
CIC $1280 \mu$ g (group A), & antibiotics therapy & antibiotics therapy \\
CIC $640 \mu$ g plus current dose of ICS (group B) or & & \\
a doubled dose of current ICS (group C) & & \\
\hline
\end{tabular}

The clinical evaluation included ACT, day and night symptoms score, rescue medication consumption, the necessity of OCS or antibiotics treatment, $A E$ and resolution of asthma exacerbation. 
Table 2. Characteristics of patients according to particular treatment options

\begin{tabular}{lccc}
\hline Parameters & Group A $(n=25)$ & Group B $(n=25)$ & Group C $(n=24)$ \\
\hline Age [years] & $44.1 \pm 13.1$ & $43.8 \pm 14.3$ & $41.6 \pm 11.9$ \\
\hline Sex F/M & $14 / 11$ & $15 / 10$ & $13 / 11$ \\
\hline Asthma history [years] & $13.6 \pm 9.6$ & $13.4 \pm 9.4$ & $16.5 \pm 10.0$ \\
\hline IgE total [IU/I] & $227.5 \pm 185.3$ & $270.2 \pm 234.3$ & $231 \pm 121.0$ \\
\hline Blood eosinophilia & $282.8 \pm 149.7$ & $273.2 \pm 148.1$ & $286.2 \pm 121.8$ \\
\hline FEV $_{1} \%$ predicted & $64.7 \pm 12.0$ & $63.4 \pm 9.2$ & $63.5 \pm 10.9$ \\
\hline $\mathrm{F}_{\text {ENO }}$ PpB & $63.2 \pm 43.2$ & $58.4 \pm 43.6$ & $60.9 \pm 36.2$ \\
\hline
\end{tabular}

Data are presented as means (SD). The differences between treatment options were not statistically significant according to Kruskal-Wallis Test.

Table 3. Changes in studied parameters between particular treatment options during the therapy period

\begin{tabular}{|c|c|c|c|c|c|}
\hline Studied parameters & Treatment days & Group A & Group B & Group C & $\begin{array}{l}\text { Differences between groups } \\
\text { day } 1 \text { vs. } 14\end{array}$ \\
\hline \multirow[t]{3}{*}{$\mathrm{FEV}_{1} \%$ predicted } & 1 & $64.7 \pm 12.0$ & $63.4 \pm 9.2$ & $63.5 \pm 10.9$ & A vs. C, $p<0.001$ \\
\hline & 7 & $75.2 \pm 12.6$ & $70.9 \pm 10.2$ & $66.7 \pm 11.6$ & A vs. $B, p=0.03$ \\
\hline & 14 & $81.4 \pm 10.9$ & $76.6 \pm 9.3$ & $71.1 \pm 9.8$ & B vs. C, $p=0.0003$ \\
\hline \multirow[t]{3}{*}{ FENO ppB } & 1 & $63.2 \pm 43.2$ & $58.4 \pm 43.6$ & $60.9 \pm 36.2$ & A vs. C, $p=0.19$ \\
\hline & 7 & $30.2 \pm 12.7$ & $32.0 \pm 11.6$ & $45.1 \pm 23.3$ & A vs. $B, p=0.36$ \\
\hline & 14 & $22.0 \pm 6.9$ & $24.6 \pm 7.2$ & $35.9 \pm 18.3$ & B vs. C, $p=0.8$ \\
\hline \multirow[t]{3}{*}{ ACT } & 1 & $6.9 \pm 1.1$ & $6.9 \pm 0.8$ & $7.1 \pm 0.7$ & A vs. C, $p<0.001$ \\
\hline & 7 & $13.6 \pm 2.3$ & $12.2 \pm 1.3$ & $9.9 \pm 1.8$ & A vs. $B, p=0.0007$ \\
\hline & 14 & $16.9 \pm 2.1$ & $14.6 \pm 1.6$ & $12.0 \pm 1.6$ & B vs. C, $p<0.001$ \\
\hline \multirow[t]{3}{*}{ Day symptoms score } & 1 & $4.2 \pm 0.4$ & $3.9 \pm 0.3$ & $3.9 \pm 0.3$ & A vs. C, $p<0.001$ \\
\hline & 7 & $2.8 \pm 0.6$ & $3.1 \pm 0.3$ & $3.8 \pm 0.4$ & A vs. $B, p=0.0023$ \\
\hline & 14 & $2.1 \pm 0.5$ & $2.5 \pm 0.5$ & $3.2 \pm 0.6$ & B vs. C, $p=0.012$ \\
\hline \multirow{3}{*}{$\begin{array}{l}\text { Night symptoms } \\
\text { score }\end{array}$} & 1 & $2.6 \pm 0.6$ & $2.7 \pm 0.5$ & $2.6 \pm 0.7$ & A vs. $C, p=0.03$ \\
\hline & 7 & $0.5 \pm 0.7$ & $1.6 \pm 0.5$ & $1.5 \pm 0.9$ & A vs. $B, p=0.045$ \\
\hline & 14 & $0.3 \pm 0.5$ & $0.6 \pm 0.6$ & $0.7 \pm 0.6$ & B vs. C, $p=0.29$ \\
\hline \multirow{3}{*}{$\begin{array}{l}\text { Rescue medication } \\
\text { consumption }\end{array}$} & 1 & $4.3 \pm 1.3$ & $4.0 \pm 1.2$ & $4.2 \pm 1.3$ & A vs. $C, p=0.02$ \\
\hline & 7 & $1.8 \pm 1.3$ & $1.8 \pm 1.1$ & $3.0 \pm 1.6$ & A vs. B, $p=0.1$ \\
\hline & 14 & $0.7 \pm 1.1$ & $0.8 \pm 0.7$ & $1.6 \pm 1.1$ & B vs. C, $p=0.04$ \\
\hline
\end{tabular}

Data are presented as means (SD). FEV - forced expiratory volume in $1 \mathrm{~s}$, FENO - exhaled nitric oxide, ACT-Asthma Control Test.

age, asthma history, laboratory results, spirometric parameters and $\mathrm{F}_{\mathrm{ENO}}$ between particular treatment options.

Both ciclesonide treatment arms have shown statistically and clinically important advantages in comparison to the group where medium doses of previously used inhaled corticosteroid were doubled. Data are presented in Table 3 and in Figures 1-4.

On day 7 and 14 of the treatment we observed statistically significant improvement of $\mathrm{FEV}_{1}$ among all groups, however, the change in the group treated with high doses of ciclesonide (group A) was significantly higher and appeared earlier than in other study groups (Table 3, Figure 1).

A decrease in $F_{\text {ENO }}$ value between baseline and day 14 was statistically significant for all treatment options.
However, the differences between the studied groups of patients were not statistically significant. The dynamics of the decrease in $F_{E N O}$ for both groups treated with ciclesonide (group A and B) were statistically significantly higher over the use of a high dose of non-extra fine ICS. Both $F_{E N O}$ decrease and its dynamics were statistically correlated with eosinophilia, IgE and presence of atopy. Higher eosinophilia, IgE and in case of atopy, a higher baseline and higher decrease in $\mathrm{F}_{\mathrm{ENO}}$ during the therapy was observed (Table 3, Figure 2).

The average value of Asthma Control Test (ACT) for particular treatment days is presented in Figure 3 and in Table 3. All three evaluated treatment methods significantly improved the degree of asthma control in comparison to base- 


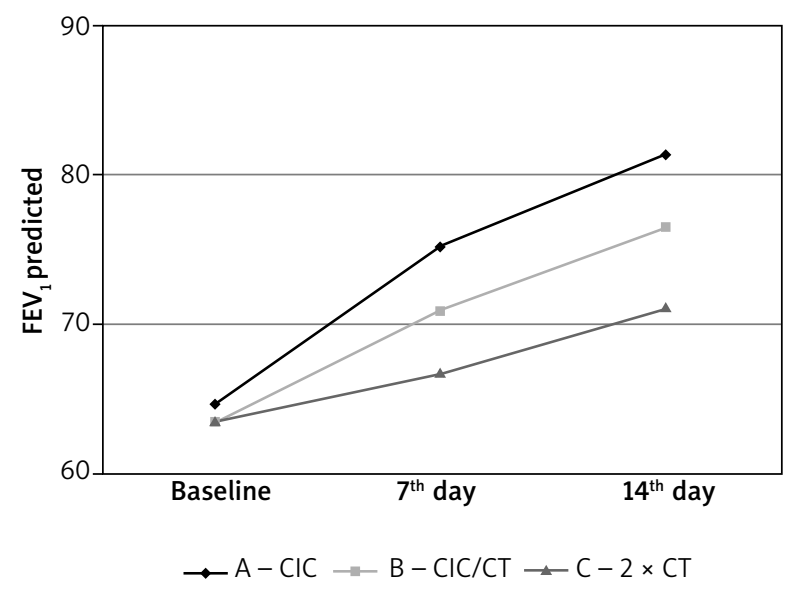

Figure 1. Changes in FEV during the study depending on particular treatment options

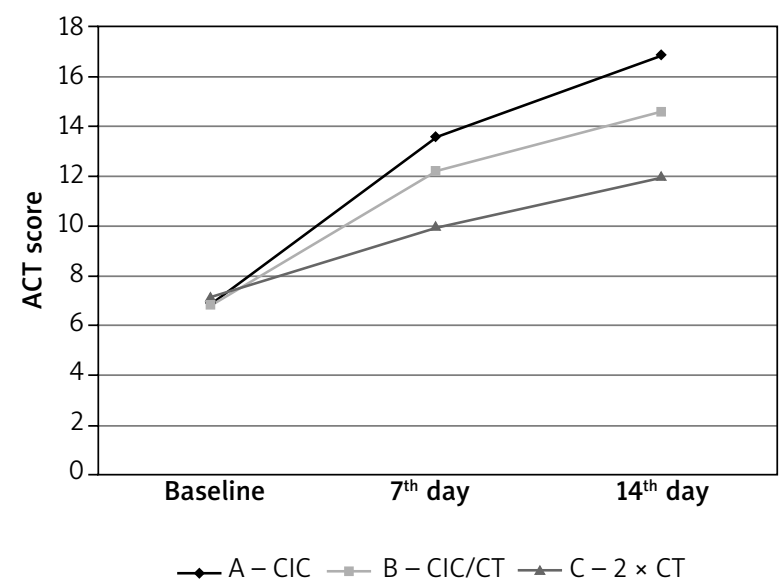

Figure 3. Changes in Asthma Control Test score during the study

line. Patients treated with high doses of ciclesonide (group A) showed a statistically higher and more dynamic increase in their ACT score (Table 3, Figure 3). All treatment strategies caused a significant reduction in rescue medication consumption. Treatment options with ciclesonide (group $A$ and $B$ ) showed significant therapeutic advantages over using a high dose of non-extra fine ICS (Table 3, Figure 4).

At the baseline we did not observe statistically significant differences in day and night symptoms score between particular treatment options. On day 7 and 14 of treatment we observed a significantly higher reduction in daytime symptoms score for both treatment arms with ciclesonide in comparison to the one using a high dose of non-extra fine inhaled corticosteroids. Night-time symptoms scores both on day 7 and 14 were statistically significantly lower in both CIC treatment arms (Table 3).

A comparison of groups $A$ and $B$ shows a greater response among patients treated with higher doses of ciclesonide (group A) (Table 3).

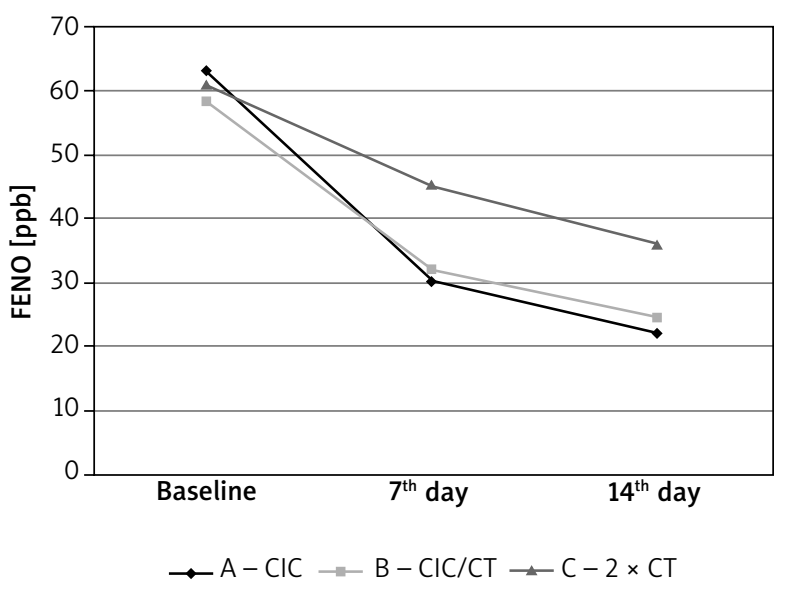

Figure 2. Changes in exhaled nitric oxide concentrations during therapy depending on selected treatment methods

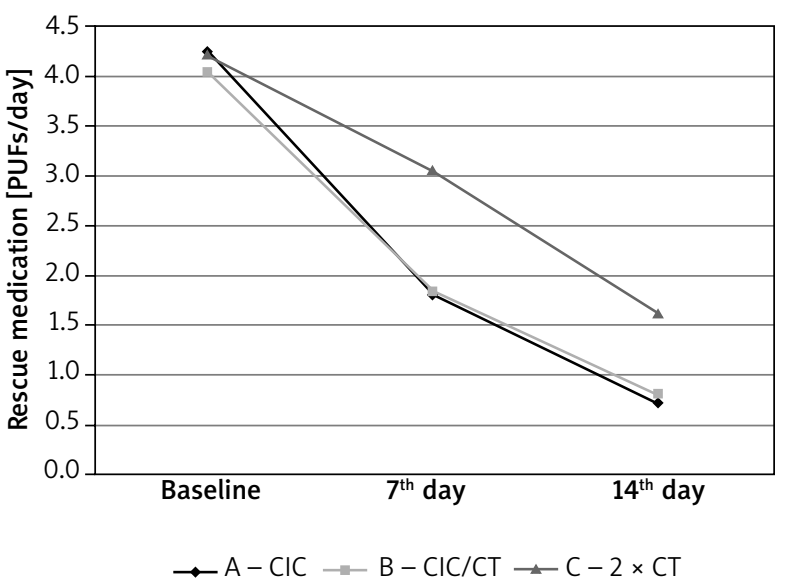

Figure 4. Changes in rescue medication consumption during the treatment period

We evaluated how often patients (depending on particular therapeutic options) required antibiotics or oral steroid treatment, adverse events frequency (connected with inhaled corticosteroid treatment) and the number of patients whose exacerbation/loss of control had been resolved after 14 days of therapy.

Patients in groups treated with ciclesonide presented statistically significant fewer cases where OCS or antibiotic therapy were necessary. Moreover, asthma exacerbation time among these patients was significantly shorter. An analysis of the endpoints of this study has been presented in Table 4.

\section{Discussion}

Small airways play a significant role in unstable and severe asthma pathogenesis [14-16]. Extra-fine ICS seem to penetrate to peripheral airways and present a more potent anti-inflammatory effect in patients with small 
Table 4. The analysis of the endpoints of this study

\begin{tabular}{lccc}
\hline Parameter & Group A $(n=25)$ & Group B $(n=25)$ & Group C $(n=24)$ \\
\hline Antibiotics treatment & 3 & 5 & 6 \\
\hline OCS treatment & 0 & 1 & 5 \\
\hline Adverse events & 0 & 1 & 4 \\
\hline End of exacerbation & 21 & 19 & 6
\end{tabular}

Data are presented as the number of cases in particular treatment group. OCS - oral corticosteroids.

airways involved. Extra-fine ICSs are effective in achieving asthma control and present an effect which is no worse than adding LABA to therapy [6].

Despite a significant progress in diagnostics and treatment of asthmatic patients, exacerbations may occur as a natural course of the disease. Progressing deterioration of lung function, dyspnoea, cough, wheezing and chest tightness are the main features of asthma exacerbation. The first step in prevention of severe asthma exacerbations is intensifying the anti-inflammatory treatment with high doses of ICS [11].

The aim of the study was to assess the efficacy of extra-fine ciclesonide in patients losing control of their asthma despite being treated with medium doses of standard size-particle ICS and LABA as the second controller.

Treating with high doses of ciclesonide brought a quick and potent clinical improvement along with the proper safety profile in patients suffering from mild asthma exacerbation (or who had been losing asthma stability). Therapeutic options of using ciclesonide (especially in high doses) showed a significant therapeutic advantage over using high doses of non-extra fine ICS. High doses of extra-fine ciclesonide allowed decreasing the need for interventional OCS therapy or/and antibiotics without additional safety concerns. Treatment with small-particle ICS (CIC) gives the opportunity to avoid the development of full-scale exacerbation (in clinical picture) and the need for OCS short-term therapies.

A possible reason for the strong anti-inflammatory effect of ciclesonide may be the solution formulation for HFA-powered MDI with a small particle size that extends and reaches the peripheral airways, which are important sites of significant inflammation in asthma patients [17]. Ciclesonide also has low oropharyngeal and high pulmonary deposition resulting in a high therapeutic index and low potential for adverse events [18]. Activation of a potent metabolite with a high affinity for pulmonary glucocorticoid receptors which provides anti-inflammatory activity at the desired target site is also very important [19]. Fatty acid conjugation of the active metabolite results in a depot effect that prolongs the anti-inflammatory action [20].

The safety of ICS, especially when using high doses, depends on their dose, formulation and pharmacological properties. Marczak published a study in which he showed that switching from prednisone to very high doses of ciclesonide normalized the hypothalamic-pituitary adrenal axis function and also improved the disease control and the lung function in patients with difficult asthma [21].

Extra-fine ICS updosing - adjustment should be a treatment of first choice in achieving satisfactory asthma control and prevention of the imminent exacerbation in practice. It is connected with anti-inflammatory properties and the ability to penetrate to small airways affected by the inflammatory process.

The possible limitations of the study are small groups of patients and a lack of randomization.

\section{Conclusions}

The original aspect of the study is to show that ciclesonide, which is safe and effective in asthma treatment, also presents a rapid clinical improvement and a potent anti-inflammatory effect in comparison to non-extra fine ICS in patients with mild asthma exacerbation/loss of control. There is an interesting strategy to apply two inhaled corticosteroids having different pharmacological properties and different deposition into the airways at the same time.

More clinical studies for assessing the long-term efficacy of ciclesonide in comparison with non-extra fine inhaled corticosteroids in preventing asthma exacerbations and maintaining asthma control are needed.

\section{Conflict of interest}

Ziemowit Zietkowski and Anna Bodzenta-Lukaszyk have received payments for lectures from Takeda and AstraZeneca. The other authors declare that they have no conflict of interest in the publication of this manuscript. This work was supported by research grant No 3-35523P from the Medical University of Bialystok, Poland.

\section{References}

1. Contoli M, Bousquet J, Fabbri LM, et al. The small airways and distal lung compartment in asthma and COPD: a time for reappraisal. Allergy 2010; 65: 141-51.

2. Grainge CL, Lau LC, Ward JA, et al. Effect of bronchoconstriction on airway remodeling in asthma. N Engl J Med 2011; 364: 2006-15. 
3. Gonem S, Natarajan S, Desai D, et al. Clinical significance of small airway obstruction markers in patients with asthma. Clin Exp Allergy 2014; 44: 499-507.

4. Bjermer $L$. The role of small airway disease in asthma. Curr Opin Pulm Med 2014; 20: 23-30.

5. Zietkowski Z, Bodzenta-tukaszyk A, Tomasiak MM, et al. Effect of ciclesonide and fluticasone on exhaled nitric oxide in patients with mild allergic asthma. Respir Med 2006; 100: 1651-6.

6. van Aalderen WM, Grigg J, Guilbert TW, et al. small-particle inhaled corticosteroid as first-line or step-up controller therapy in childhood asthma. J Allergy Clin Immunol Pract 2015; 3: 721-31.

7. Israel E, Roche N, Martin RJ, et al. Increased dose of inhaled corticosteroid versus add-on long-acting beta-agonist for step-up therapy in asthma. Ann Am Thorac Soc 2015; 12: 798-806.

8. Perez T, Chanez P, Dusser D, Devillier P. Small airway impairment in moderate to severe asthmatics without significant proximal airway obstruction. Respir Med 2013; 107: 1667-74.

9. Van den Berge M, ten Hacken NHT, van der Wiel E, Postma DS. Treatment of the bronchial tree from beginning to end: targeting small airway inflammation in asthma. Allergy 2013; 68: 16-26.

10. Cohen J, Douma WR, ten Hacken NHT, et al. Ciclesonide improves measures of small airway involvement in asthma. Eur Respir J 2008; 31: 1213-20.

11. Global Initiative for Asthma. Global strategy for asthma management and prevention (updated 2014). http:// www. ginaasthma.org.

12. American Thoracic Society/American Lung Association Recommendations for On-line Measurement of Exhaled Nitric Oxide in Adults and the Recommendations for On-line, Offline and Nasal Expired Nitric Oxide Measurements in Children. Am J Respir Crit Care Med 1999; 160: 2104-17.

13. American Thoracic Society. Lung function testing: selection of reference values and interpretative strategies. Am Rev Respir Dis 1991; 144: 1202-18.

14. Thompson BR, Douglass JA, Ellis MJ, et al. Peripheral lung function in patients with stable and unstable asthma. J Allergy Clin Immunol 2013; 131: 1322-8.

15. Veen JC, Beekman AJ, Bel EH, Sterk PJ. Recurrent exacerbations in severe asthma are associated with enhanced airway closure during stable episodes. Am J Respir Crit Care Med 2000; 161: 1902-6.

16. Bourdin A, Paqanin F, Prefaut C, et al. Nitrogen washout slope in poorly controlled asthma. Allergy 2006; 61: 85-9.

17. Thompson PJ. Drug delivery to the small airways. Am J Respir Crit Care Med 1998; 157: 199-202.

18. Rohatagi S, Derendorf H, Zech K, et al. PK/PD of inhaled corticosteroids: the risk/benefit of inhaled ciclesonide. J Allergy Clin Immunol 2003; 111: A598.

19. Kelly HW. Pharmaceutical characteristics that influence the clinical efficacy of inhaled corticosteroids. Ann Allergy Asthma Immunol 2003; 91: 326-34.

20. Nave R, Sattele N, Mayer W. Formation of fatty acid conjugates of ciclesonide-active principle in the rat lung after 4-week inhalation of ciclesonide. Am J Respir Crit Care Med 2003; 167: A1509.

21. Marczak J, Ciebiada M, Górski P. Switching from systemic steroids to ciclesonide restores the hypothalamic pituitaryadrenal axis. Adv Dermatol Allergol 2014; 31: 59-64. 